\title{
In Vitro and In Silico Studies of Quercetin and Daidzin as Selective Anticancer Agents
}

\author{
Muhammad Sulaiman Zubair $^{1^{*}}$, Syariful Anam ${ }^{1}$, Saipul Maulana ${ }^{1}$, and Muhammad Arba ${ }^{2}$ \\ ${ }^{1}$ Department of Pharmacy, Faculty of Mathematics and Natural Sciences, Tadulako University, Palu 94118, Indonesia \\ ${ }^{2}$ Department of Pharmacy, Faculty of Pharmacy, Universitas Halu Oleo, Kendari 93231, Indonesia
}

\section{* Corresponding author:}

email: sulaiman_zubair80@yahoo.co.id

Received: January 19, 2020

Accepted: November 2, 2020

DOI: $10.22146 /$ ijc. 53552

\begin{abstract}
Quercetin and daidzin are flavonoid and flavonoid glycoside type compounds that have been found in many plants and nutraceuticals. This study aims to examine the in vitro cytotoxic and selectivity properties of quercetin and daidzin on breast and cervical cancers and to study their molecular interaction and stability on epidermal growth factor receptor tyrosine kinase (EGFR-TK) by applying molecular docking and molecular dynamics $(M D)$ simulations. In vitro anticancer activity was performed by 3-(4,5dimethylthiazol-2-yl)-2,5-diphenyltetrazolium bromide (MTT) method on breast cancer cell (T47D), cervical cancer cells (HeLa), and Vero normal cells, while molecular docking and MD simulation were done by using AutoDock Vina and Amber18 package software, respectively. Quercetin and daidzin showed potent cytotoxic and high selectivity on both cell lines. Daidzin was found to has a higher $I C_{50}$ and selectivity index than quercetin. Docking and MD results showed that both compounds prefer to interact with epidermal growth factor receptor tyrosine kinase (EGFR-TK). Daidzin showed better interaction than quercetin with a docking score of $-9.6 \mathrm{kcal} / \mathrm{mol}$. Also, daidzin was found more stable than quercetin with low RMSD and RMSF values.
\end{abstract}

Keywords: quercetin; daidzin; T47D; HeLa; docking; molecular dynamics

\section{- INTRODUCTION}

In recent times, computational applications for predicting the molecular interactions on the biological system are broadly used to study molecular recognition or design more potent bioactive compounds. Molecular docking and molecular dynamics are two kinds of recent computational methods that can provide molecular interactions between receptor proteins and drugs and how they stabilize the interaction. These methods focused on protein-drugs (ligands) complexes to study how the drugs bind to the protein receptor, which is very important for discovering or developing new bioactive compounds in fighting against diseases. Besides, the methods for identification of protein-ligand interaction fingerprints that featuring how strong the interaction has developed as well [1-2].

In terms of cancer diseases, many molecular mechanisms of cell proliferation inhibition have been reported so far. The advanced technique of molecular assay on cancer research has led to the increasing knowledge of the molecular basis of carcinogenesis that opens the possibility of discovering a more selective chemopreventive agent, mostly from the natural product. Attention has been focused on natural substances capable of inhibiting carcinogenesis via the apoptosis mechanism [3].

Quercetin and daidzin (Fig. 1) are two natural anticancer compounds that have been given much attention at this time. Quercetin is a flavonoid found mainly in various vegetables and fruits, such as capers, lovage, dill, cilantro, and onions. It was reported to suppress the HeLa cells by blocking the phosphatidylinositol 3-kinase (PI3K)-Akt/PKB (protein kinase B) pathway. Quercetin also induces apoptosis by activation of intrinsic apoptotic pathway associated with upregulation of Bax, Bad, Bid, caspase-9, -3, downregulation of $\mathrm{Bcl}-2 ; \mathrm{Bcl}-\mathrm{xL}$, and cytochrome $\mathrm{c}$ release and inhibit NF- $\kappa B, P K C-\delta$, ERK1/2 along with 

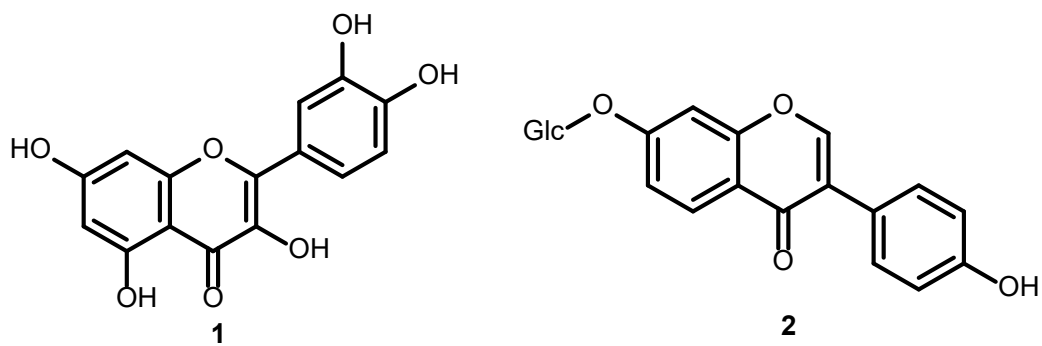

Fig 1. Quercetin (1) and daidzin (2)

AMPK activation and downregulation of uPA/uPAR, MMP-9, and -2 for cell inhibition of migration and invasion [4-7]. Meanwhile, daidzin, an ingredient of soy isoflavones, is a glycoside form of daidzin flavonoid. It has also been reported to possess anticancer activity in early prostate cancer development stages and reduce postmenopausal breast cancer risk. It can inhibit telomerase activity by hydrogen bonding with the base of G-quadruplex as well [8-10].

To obtain more insight into the selectivity and stability properties of the anticancer mechanism of quercetin and daidzin, in this present study, they were in vitro tested on breast and cervical cancers along with the normal cells and in silico molecular docking, and molecular dynamics on epidermal growth factor receptor tyrosine kinase (EGFR-TK) were also performed. EGFRTK has been known as a target for flavonoids and flavonoid glycoside on their activity in inhibiting cancer cell proliferation and transformation [11]. This study is the first report regarding the molecular docking and MD simulations of quercetin and daidzin on EGFR-TK.

\section{- EXPERIMENTAL SECTION}

\section{Materials}

Quercetin and daidzin were purchased from Sigma Aldrich. RPMI-1640, Streptomycin, Penicillin, Fetal Bovine Serum (FBS), Dimethylsulphoxide (DMSO), and MTT were purchased from Merck.

Human ductal breast epithelial tumor cell line (T47D), human cervical cancer cell line (HeLa), and green African monkey renal epithelial cell (Vero) were obtained from Laboratory of Parasitology, Faculty of Medicine, Universitas Gadjah Mada. Cells were maintained in RPMI-1640 medium supplemented with $100 \mu \mathrm{g} / \mathrm{mL}$ streptomycin, 100 units $/ \mathrm{mL}$ penicillin, and $10 \%$ fetal bovine serum (FBS) in $5 \% \mathrm{CO}_{2}$ atmosphere at $37^{\circ} \mathrm{C}$.

\section{Procedure}

\section{Cytotoxicity test}

Cytotoxic activity was applied to the Human ductal breast epithelial tumor cell line (T47D), human cervical cancer cell line (HeLa), and green African monkey renal epithelial cell (Vero) cell lines by the MTT method as described in our previous study [12]. The stock samples were briefly diluted with RPMI-1640 medium to desired concentrations of $25,12.5,6.25,3.125,1.5625,0.7812$, and $0.3906 \mu \mathrm{g} / \mathrm{mL}$. The final concentration of dimethylsulphoxide (DMSO) in each sample was $1 \%$ $\mathrm{v} / \mathrm{v}$. The cancer cells were batch cultured for 10 days, then seeded in 96 well plates of $1 \times 10^{4}$ cells/well in fresh complete growth medium in 96-well microtiter plastic plates at $37{ }^{\circ} \mathrm{C}$ for $24 \mathrm{~h}$ under $5 \% \mathrm{CO}_{2}$ using a waterjacketed carbon dioxide incubator (CelCulture, Esco Medical ApS, Denmark). The medium (without serum) was added, and cells were incubated either alone (negative control) or with different sample concentrations. After $48 \mathrm{~h}$ of incubation, cells were added with $10 \mu \mathrm{L} /$ well of MTT $(5 \mathrm{mg} / \mathrm{mL})$ and incubated for $4 \mathrm{~h}$ in an incubator at $37^{\circ} \mathrm{C}$ in a $5 \% \mathrm{CO}_{2}$ humidified atmosphere. The reaction was stopped by $100 \mu \mathrm{L}$ dimethylsulfoxide (DMSO). The plate was then incubated for $15 \mathrm{~min}$. Each well's absorbance was read at $550 \mathrm{~nm}$ wavelength in Elisa Reader (Infinite M200 pro NanoQuant, Tecan, Switzerland), using wells without cells as blanks. All experiments were performed in triplicate. The effect of compounds on the proliferation of cancer cells was expressed as the \% cytoviability, using the following formula: 
$\%$ Cytoviability $=\frac{\text { Absorbance of treated cells }}{\text { Absorbance of controll cells }} \times 100 \%$

The $\mathrm{IC}_{50}$ calculation was done statistically by probit analysis using SPSS 17.0 (SPSS Inc., Chicago IL, USA), in which the series of dose-response data and the percentage of cytoviability were plotted together.

\section{Molecular docking}

Molecular docking was performed using AutoDock Vina [13]. EGFR-TK protein target was obtained from the Brookhaven Protein Data Bank (www.rscb.org) with the code 1M17. BIOVIA Discovery Studio 2017 was used to separate water, native ligand, and other non-standard residues and visualizes the docking result [14]. AutoDock Tools (ADT) was used to optimize and prepare the required files to be docked. The grid spacing of $0.375 \AA$ and the grid points in $\mathrm{X}, \mathrm{Y}$, and Z-axis were set at $42 \times 40$ $\times 40$ points. The grid center coordinates were placed at $X$ : 21.697, Y: 0.303, and Z: 52.093. ChemDraw (ACDLabs, Netherland) was used to build quercetin and daidzin chemical structures and converted to the $3 \mathrm{D}$ structure after optimization using the MM2 method. Docking methods and parameters were validated by redocking the co-crystallized ligands erlotinib into the protein structure. The root-mean-square deviation (RMSD) value was calculated using the rms_cur module in PyMol [15]. The default settings of each software were used if no further explanation. Docking was performed on Linux operating system with Intel(R) Core ${ }^{\mathrm{rm}} 2$ Duo CPU T5800@ 2 GHz as a processor and $1.93 \mathrm{~GB}$ of RAM.

\section{Molecular dynamics}

Molecular dynamics (MD) simulation was carried out for quercetin and daidzin on EGFR-TK employing the AMBER18 package [16]. Leap module was used to prepare each complex using the ff99SB force field for protein and GAFF force field and AM1-BCC for ligands [17-19]. Counterions were added to the neutralized complex. Each complex was placed inside a truncated octahedron TIP3P water model with a $12 \AA$ distance between the complex and the box's edge. Each complex underwent two-step minimization. In the first step, minimization was performed for water and ions while protein-ligand was restrained with a $500 \mathrm{kcal} \mathrm{mol}^{-1} \AA^{-2}$ and to heat the system ligand-protein in $300 \mathrm{~K}$. Next, only backbone atoms of the protein were restrained with a force constant of $500 \mathrm{kcal} \mathrm{mol}^{-1} \AA^{-2}$.

Further, following the minimization step, each complex underwent a heating step for 120 ps with 1000 steps minimization, and backbone atoms of protein were restrained with a force constant of $500 \mathrm{kcal} / \mathrm{mol} \AA^{-2}$. The system was then equilibrated at $300 \mathrm{~K}$, and the density was $1 \mathrm{~g} / \mathrm{mL}$ for 60.000 steps each with a 2 fs time step giving simulation lengths of $120 \mathrm{ps}$. The production MD simulation was performed for $8 \mathrm{~ns}$ in the NPT ensemble without any restraint employing PMEMD (Particle Mesh Ewald Molecular Dynamics). The Cuda module under a periodic boundary condition with a nonbonding cutoff distance of $12.0 \AA$. The SHAKE algorithm was used to constrain all bonds involving hydrogen atoms with a 2 fs integration time step [20]. The particle-mesh Ewald algorithm method was used to treat long-range electrostatic interactions of a periodic box with a non-bonding cutoff distance of $12.0 \AA$ [21]. The Langevin thermostat was used to control the Langevin thermostat with a collision rate of $1.0 \mathrm{ps}^{-1}$. The coordinate files were saved every 1 ps. The Root Mean Square Deviation (RMSD) and Root Mean Square Fluctuation (RMSF) were analyzed with the CPPTRAJ module of AMBER18 [22], while visualization and hydrogen bond occupancy in the protein-ligand system was conducted using the Visual Molecular Dynamics (VMD) software [23].

\section{- RESULTS AND DISCUSSION}

\section{In Vitro Cytotoxicity}

Cytotoxic activity of quercetin and daidzin was tested on the breast (T47D) and cervical (HeLa) cancer cell lines. It was found that daidzin has more potent cytotoxic activity than quercetin on T47D and HeLa cell lines with the $\mathrm{IC}_{50}$ of 0.04 and $0.54 \mu \mathrm{g} / \mathrm{mL}$, respectively. Moreover, daidzin did not toxic on Vero cell lines (normal cells) at the concentration of $100 \mu \mathrm{g} / \mathrm{mL}$. Meanwhile, quercetin showed $\mathrm{IC}_{50}$ of $41.57 \mu \mathrm{g} / \mathrm{mL}$ on Vero cell lines. Therefore, the selectivity index can be calculated by the formula; $\mathrm{IC}_{50}$ of a compound on Vero cells/ $\mathrm{IC}_{50}$ of a compound on cancer cells. Selectivity index $>3$ can be categorized as having high selectivity [24]. 
Table 1. Cytotoxic activity of quercetin and daidzin against cancer cell lines

\begin{tabular}{lccccc}
\hline Compounds & \multicolumn{3}{c}{$\mathrm{IC}_{50}(\mu \mathrm{g} / \mathrm{mL})$} & \multicolumn{2}{c}{ Selectivity Index $(\mathrm{SI})$} \\
\cline { 2 - 6 } & $\mathrm{T} 47 \mathrm{D}$ & $\mathrm{HeLa}$ & Vero & $\mathrm{T} 47 \mathrm{D}$ & $\mathrm{HeLa}$ \\
\hline Quercetin & $9.58 \pm 2.34$ & $3.66 \pm 0.84$ & $41.57 \pm 26.33$ & 4.34 & 11.36 \\
Daidzin & $0.04 \pm 0.04$ & $0.54 \pm 0.07$ & $>100(\mathrm{NT})$ & $\mathrm{S}$ & $\mathrm{S}$ \\
\hline
\end{tabular}

NT: No Toxicity at $100 \mu \mathrm{g} / \mathrm{mL}, \mathrm{S}$ : Selective (High selectivity index, SI > 3.0)

Based on this, daidzin can be considered to have high selectivity. Meanwhile, quercetin showed to be more selective on the HeLa cell than the T47D cell (Table 1).

The different anticancer activity and selectivity properties of quercetin and daidzin might be caused by glucose unit presence, forming a glycosidic bond with the daidzin hydroxyl group [25]. Daidzin was reported as a potent tyrosinase inhibitor based on the glycoside moieties, and mostly the glycoside form of flavonoids was absorbed in the small intestine [26-27].

\section{Molecular Docking and Molecular Dynamics}

Molecular docking and molecular dynamics of both compounds were performed on epidermal growth factor receptor tyrosine kinase (EGFR-TK) responsible for breast and cervical cancer cell proliferation. EGFR (also known as Erb-B1 or HER-1) is a tyrosine kinase receptor that able to activate different signaling cascades involved in the pathogenesis of carcinomas, such as phosphatidylinositol 3-kinase (PI3K)/Akt and mitogenactivated protein kinase (MAPK) [28]. Docking protocol was validated by redocking native ligand erlotinib to EGFR-TK receptors (PDB Id: 1M17). It can be seen from Table 2, that native ligand erlotinib has an RMSD value < $2 \AA$, which means the protocol used has prospectively validated [29-30]. It is interesting to note that daidzin showed good interaction on the EGFR-TK receptor target that might be responsible for the high cytotoxicity on cancer cells. Daidzin has lower docking energy on epidermal growth factor receptor - tyrosine kinase (EGFR-TK) proteins than quercetin, indicating a higher binding affinity of daidzin compared to quercetin.

Quercetin was found to interact with the catalytic residue of MET769 by hydrogen bonding and showed hydrophobic interaction with several amino acids of LEU 820, LEU694, ALA719, VAL702, and LYS721 in the hydrophobic pocket of EGFR-TK protein. Meanwhile,
Table 2. Docking score of quercetin and daidzin on EGFR-TK receptor target

\begin{tabular}{lcc}
\hline Compounds & $\begin{array}{c}\text { Docking Energy } \\
(\text { Kcal/mol })\end{array}$ & $\begin{array}{c}\text { RMSD } \\
(\AA)\end{array}$ \\
\hline Quercetin & -9.0 & - \\
Daidzin & -9.6 & - \\
Native Ligand Erlotinib & -7.1 & 1.912 \\
\hline
\end{tabular}

daidzin did not show that kind of interactions where the hydrogen bonding was found only between the glycoside group and amino acid residues of ALA719, LYS721, LEU764, THR830, and ASP831 located on the EGFR phosphate-binding region along with the sugar pocket. Besides, the aglycone part of daidzin also showed hydrophobic interaction with amino acids of LEU820, VAL702, and LEU694 (Fig. 2).

Further study was carried out by employing molecular dynamics (MD) simulation to investigate the stability of the interaction between quercetin and daidzin on the EGFR-TK binding pocket. Based on quercetin and daidzin are well-known natural compounds with anticancer activity, the MD simulation was done for $8 \mathrm{~ns}$ [31-33]. Root mean square deviation (RMSD) value was used to monitor interaction stability (Fig. 3). It can be seen that quercetin, daidzin, and native ligand erlotinib maintained stable interaction on the EGFR-TK binding pocket with an overall RMSD value lower than $3 \AA$. However, quercetin (blue) was found to have a high RMSD value ( $>3 \AA$ ) at the end of the simulation, indicating less stability of quercetin than daidzin and native ligand erlotinib during $8 \mathrm{~ns}$ of simulation time.

Further evaluation of the fluctuation of amino acid residues of EGFR-TK during the dynamics run can be seen on the root mean square fluctuation (RMSF) graph (Fig. 4). A similar pattern of amino acid fluctuation was seen in the whole region of EGFR-TK, indicating a 

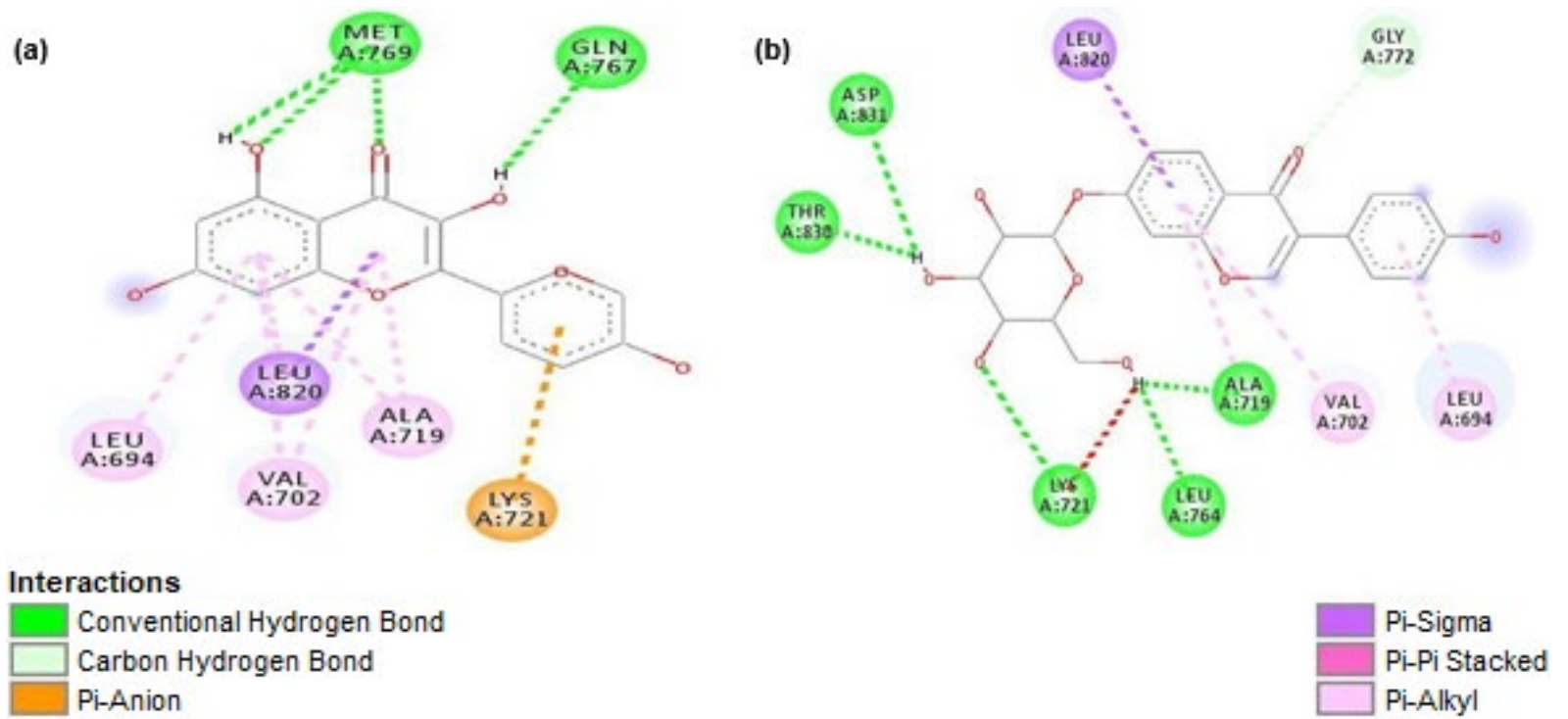

Fig 2. Molecular interaction between quercetin (a), and daidzin (b) on active site of EGFR-TK (pdb code 1M17)

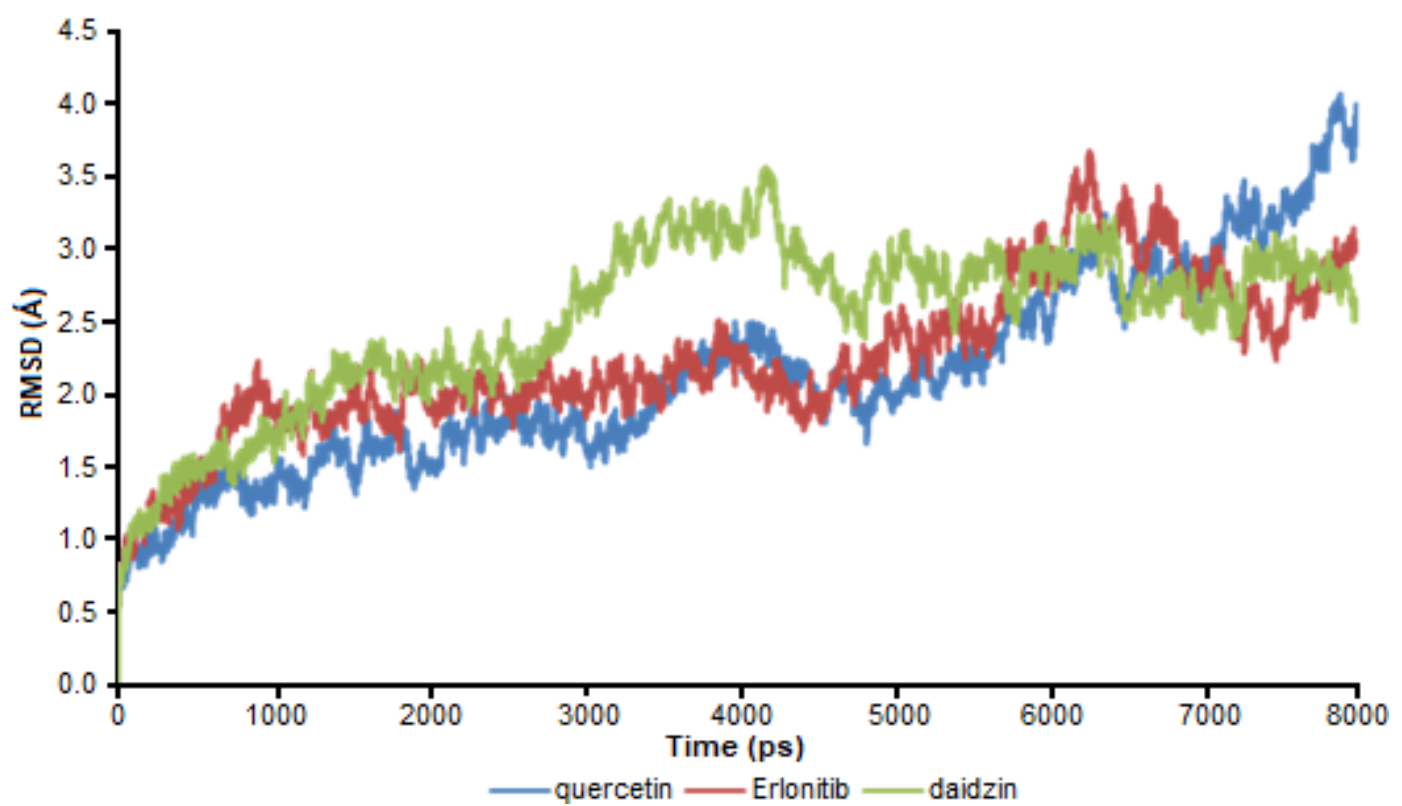

Fig 3. RMSD value of ligands on EGFR-TK during $8 \mathrm{~ns}$ MD calculated for quercetin (blue), Daidzin (green), and native ligand erlotinib (red)

similar pattern of binding interaction of native ligand erlotinib, quercetin, and daidzin. However, quercetin was found to have a higher RMSF value than daidzin and native ligand erlotinib, indicating that quercetin more fluctuated during $8 \mathrm{~ns} \mathrm{MD}$ simulation, affording the less stable interaction. Hydrogen bond interactions of docked conformations of quercetin and daidzin were not observed after $8 \mathrm{~ns}$ MD simulation, indicating that the most stable interaction was caused by van der Waals and hydrophobic type interactions.

From this study, molecular docking and molecular dynamics of quercetin and daidzin showed that epidermal growth factor receptors tyrosine kinase (EGFR-TK) was the potential target of the compounds, and this target was well-marked to have a contribution to the development and malignancy of breast cancer and 


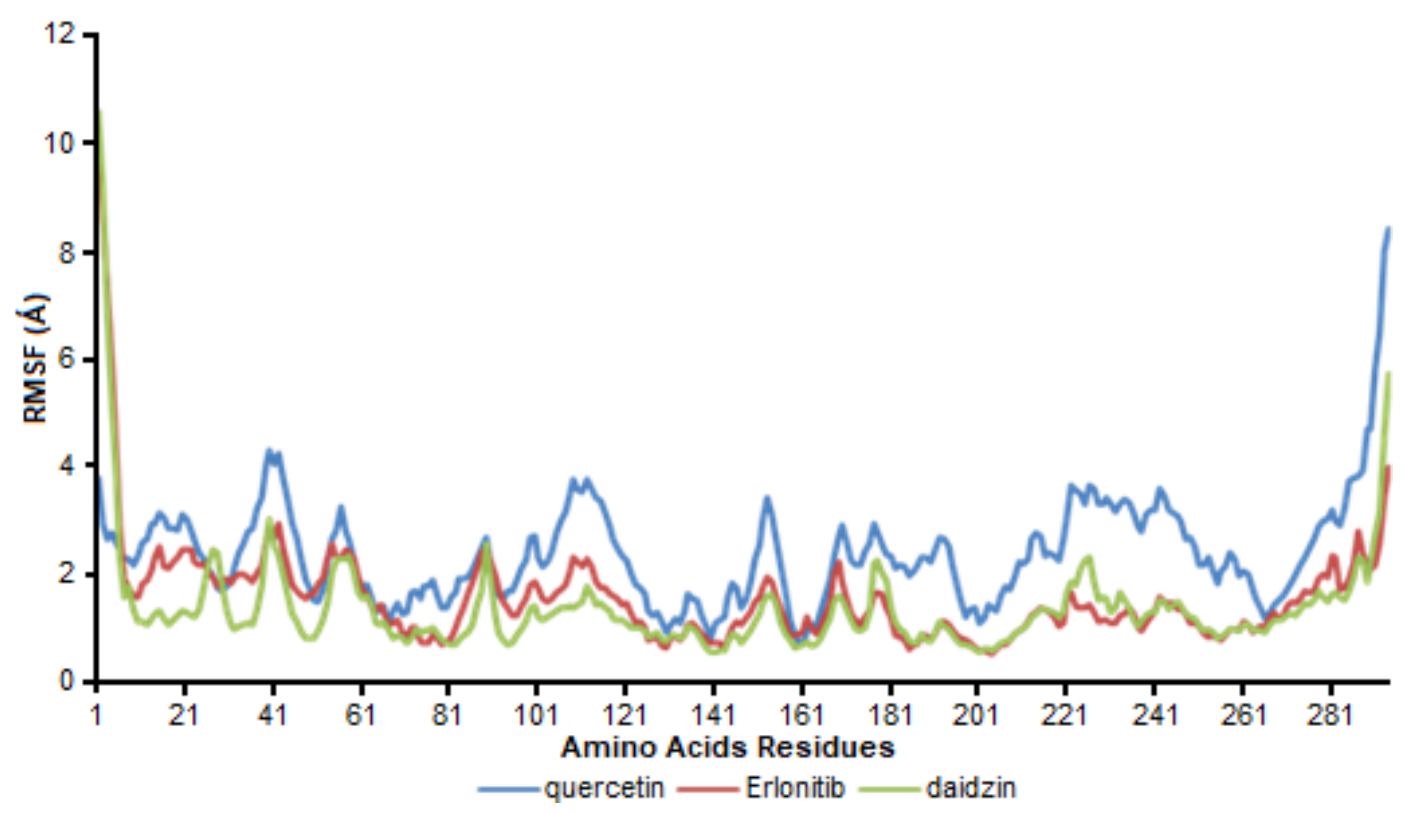

Fig 4. RMSF plotting during $8 \mathrm{~ns}$ MD simulation for quercetin (blue), daidzin (green), and native ligand erlotinib (red)

cervical cancers [34-35]. Daidzin is suggested to be a chemical scaffold for the development of epidermal growth factor receptors tyrosine kinase (EGFR-TK) protein inhibitor based on the high cytotoxicity, selectivity, and stability properties.

\section{- CONCLUSION}

In conclusion, daidzin is more cytotoxic and selective on T47D and HeLa cell lines than quercetin by in vitro assay. The presence of glucose units in daidzin might be responsible for this activity. The docking result showed that daidzin has lower energy than quercetin, attributed by glucose unit in daidzin that possessing the interaction by hydrogen bonding with the amino acid residues of ALA719, LYS721, LEU764, THR830, and ASP831. Molecular dynamics simulation exhibited that daidzin also has lower RMSD and RMSF values than quercetin, indicating more stability during interaction on EGFR-TK protein in $8 \mathrm{~ns}$ MD simulation.

\section{- ACKNOWLEDGMENTS}

The authors would like to acknowledge the Ministry of Research, Technology, and Higher Education, Republic of Indonesia, for supporting this study as part of the INSINAS 2015 (RD-2015-0106) grant.

\section{- REFERENCES}

[1] Vasseur, R., Baud, S., Steffenel, L.A., Vigouroux, X., Martiny, L., Krajecki, M., and Dauchez, M., 2015, Inverse docking method for new proteins targets identification: A parallel approach, Parallel Comput., 42, 48-59.

[2] Istyastono, E.P., 2017, Binary quantitative structure-activity relationship analysis to increase the predictive ability of structure-based virtual screening campaigns targeting cyclooxygenase-2, Indones. J. Chem., 17 (2), 322-329.

[3] Phosrithong, N., and Ungwitayatorn, J., 2010, Molecular docking study on anticancer activity of plant-derived natural products, Med. Chem. Res., 19 (8), 817-835.

[4] Teekaraman, D., Elayapillai, S.P., Viswanathan, M.P., and Jagadeesan, A., 2019, Quercetin inhibits human metastatic ovarian cancer cell growth and modulates components of the intrinsic apoptotic pathway in PA-1 cell line, Chem. Biol. Interact., 300, 91-100.

[5] Li, H., and Chen, C., 2018, Quercetin has antimetastatic effects on gastric cancer cells via the interruption of $\mathrm{uPA} / \mathrm{uPAR}$ function by modulating 
NF- $\mathrm{b}, \mathrm{PKC}-\delta, \mathrm{ERK} 1 / 2$, and AMPKa, Integr. Cancer Ther., 17 (2), 511-523.

[6] Khan, F., Niaz, K., Maqbool, F., Hassan, F.I., Abdollahi, M., Venkata, K.C.N., Nabavi, S.M., and Bishayee, A., 2016, Molecular targets underlying the anticancer effects of quercetin: An update, Nutriens, 8 (9), 529.

[7] Xiang, T., Fang, Y., and Wang, S.X., 2014, Quercetin suppresses HeLa cells by blocking PI3K/Akt pathway, J. Huazhong Univ. Sci. Technol., Med. Sci., 34 (5), 740-744.

[8] Wang, Q., Ge, X., Tian, X., Zhang, Y., Zhang, J., and Zhang, P., 2013, Soy isoflavone: The multipurpose phytochemical (Review), Biomed. Rep., 1 (5), 697-701.

[9] Boucher, B.A., Cotterchio, M., Anderson, L.N., Kreiger, N., Kirsh, V.A., and Thompson, L.U., 2012, Use of isoflavone supplements is associated with reduced postmenopausal breast cancer risk, Int. J. Cancer, 132, 1439-1450.

[10] Shan, C., Tan, J.H., Ou, T.M., and Huang, Z.S., 2013, Natural products and their derivatives as Gquadruplex binding ligands, Sci. China Chem., 56 (10), 1351-1363.

[11] Chen, H., Yao, K., Nadas, J., Bode, A.M., Malakhova, M., Oi, N., Li, H., Lubet, R.A., and Dong, Z., 2012, Prediction of molecular targets of cancer preventing flavonoid compounds using computational methods, PLoS ONE, 7 (5), e38261.

[12] Zubair, M.S., Anam, S., and Lallo, S., 2016, Cytotoxic activity and phytochemical standardization of Lunasia amara Blanco wood extract, Asian Pac. J. Trop. Biomed., 6 (11), 962-966.

[13] Trott, O., and Olson, A.J., 2010, AutoDock Vina: Improving the speed and accuracy of docking with a new scoring function, efficient optimization and multithreading, J. Comput. Chem., 31 (2), 455-461.

[14] Dassault Systèmes BIOVIA, 2016, Discovery Studio Modeling Environment Release 2017, Dassault Systèmes, San Diego, USA.

[15] Seeliger, D., and de Groot, B.L., 2010. Ligand docking and binding site analysis with PyMOL and Autodock/Vina, J. Comput.-Aided Mol. Des., 24 (5), 417-422.
[16] Arba, M., Ruslin, Kalsum, W.U., Alroem, A., Muzakkar, M.Z., Usman, I., and Tjahjono, D.H., 2018, QSAR, molecular docking and dynamics studies of quinazoline derivatives as inhibitor of phosphatidylinositol 3-kinase, J. Appl. Pharm. Sci., 8 (5), 1-9.

[17] Maier, J.A., Martinez, C., Kasavajhala, K., Wickstrom, L., Hauser, K.E., and Simmerling, C., 2015, ff14SB: Improving the accuracy of protein side chain and backbone parameters from ff99SB, J. Chem. Theory Comput., 11 (8), 3696-3713.

[18] Wang, J.M., Wolf, R.M., Caldwell, J.W., Kollman, P.A., and Case, D.A., 2004, Development and testing of a general amber force field, J. Comput. Chem., 25 (9), 1157-1174.

[19] Jakalian, A., Jack, D.B., and Bayly, C.I., 2002, Fast, efficient generation of high-quality atomic charges. AM1-BCC model: II. Parameterization and validation, J. Comput. Chem., 23 (16), 1623-1641.

[20] Elber, R., Ruymgaart, A.P., and Hess, B., 2011, SHAKE parallelization, Eur. Phys. J. Spec. Top., 200 (1), 211-223.

[21] Darden, T., York, D., and Pedersen, L., 1993, Particle mesh Ewald: An N.log(N) method for Ewald sums in large systems, J. Chem. Phys., 98 (12), 10089-10092.

[22] Roe, D.R., and Cheatham III, T.E., 2013, PTRAJ and CPPTRAJ: Software for processing and analysis of molecular dynamics trajectory data, J. Chem. Theory Comput., 9 (7), 3084-3095.

[23] Humphrey, W., Dalke, A., and Schulten, K., 1996, VMD: Visual molecular dynamics, J. Mol. Graphics, 14 (1), 33-38.

[24] Mahmoud, A.M., and El-Shemy, H.A., 2012, Efficacy assessment for several natural products with potential cytotoxic activity against breast and cervix cancers, J. Arid Land Stud., 22, 107-110.

[25] Kim, D.H., Jung, H.A., Park, S.J., Kim, J.M., Lee, S., Choi, J.S., Cheong, J.H., Ko, K.H., and Ryu, J.H., 2010, The effects of daidzin and its aglycon, daidzein, on the scopolamine-induced memory impairment in male mice, Arch. Pharmacal Res., 33 (10), 1685-1690. 
[26] Liu, H., Zhu, Y., Wang, T., Qi, J., and Liu, X., 2018, Enzyme-site blocking combined with optimization of molecular docking for efficient discovery of potential tyrosinase specific inhibitors from Puerariae lobatae Radix, Molecules, 23 (10), 2612.

[27] Heim, K.E., Tagliaferro, A.R., and Bobilya, D.J., 2002, Flavonoid antioxidants: Chemistry, metabolism and structure-activity relationships, J. Nutr. Biochem., 13 (10), 572-584.

[28] Wee, P., and Wang, Z., 2017, Epidermal growth factor receptor cell proliferation signaling pathways, Cancers, 9 (5), 52.

[29] Fajrin, F.A., Nugroho, A.E., Susilowati, R., and Nurrochmad, A., 2018, Molecular docking analysis of ginger active compound on transient receptor potential cation channel subfamily $\mathrm{V}$ member 1 (TRPV1), Indones. J. Chem., 18 (1), 179-185.

[30] Zubair, M.S., Anam, S., Khumaidi, A., Susanto, Y., Hidayat, M., and Ridhay, A., 2016, Molecular docking approach to identify potential anticancer compound from Begonia (Begonia sp), AIP Conf. Proc., 1755 (1), 080005.

[31] Prasasty, V.D., and Istyastono, E.P., 2020, Structurebased design and molecular dynamics simulations of pentapeptide AEYTR as a potential acetylcholinesterase inhibitor, Indones. J. Chem., 20 (4), 953-959.

[32] Liu, K., and Kokubo, H., 2017, Exploring the stability of ligand binding modes to proteins by molecular dynamics simulations: A cross-docking study, J. Chem. Inf. Model., 57 (10), 2514-2522.

[33] Arba, M., and Tjahjono, D.H., 2015, The binding modes of cationic porphyrin-anthraquinone hybrids to DNA duplexes: in silico study, J. Biomol. Struct. Dyn., 33 (3), 657-665.

[34] Masuda, H., Zhang, D., Bartholomeusz, C., Doihara, H., Hortobagyi, G.N., and Ueno, N.T., 2012, Role of epidermal growth factor receptor in breast cancer, Breast Cancer Res. Treat., 136 (2), 331-345.

[35] Viswanath, L., Naveen, T., Siddanna, P.,Chetana, P., Geethasree, M., Pasha, S.C.R.T., Bindhu, J., Pramod, K.P.R., Ashalatha, D., Priyadarshni, V., Ajai, G.V., Ashwini, V., Mahalakshmi, A., Riach, T., Sugashwaran, S., and Yeshaswini, T., 2014, Epidermal growth factor receptor (EGFR) overexpression in patients with advanced cervical cancer, J. Clin. Oncol., 321 (Suppl. 15), e16538. 